\title{
Neutral Particles at the Boundary of Microwave Discharge Plasma in HYPER-I
}

\author{
Akira TSUSHIMA, Shinji YOSHIMURA ${ }^{1)}$ and Yoshifumi SAITOU ${ }^{2)}$ \\ Yokohama National University, Yokohama 240-8501, Japan \\ ${ }^{1)}$ National Institute for Fusion Science, Toki 509-5292, Japan \\ ${ }^{2)}$ Utsunomiya University, Utsunomiya 321-8585, Japan
}

(Received 8 December 2009 / Accepted 31 March 2010)

\begin{abstract}
The first measurement of neutral gas pressure modulation in the range of $10 \mathrm{kHz}$ was performed using a piezoelectric transducer near the cylindrical inner wall surrounding microwave discharge plasma in the HYPER-I device. The signals from the transducer output were investigated with the ion saturation current signals from two Langmuir probes located at the plasma boundary layer.
\end{abstract}

(C) 2010 The Japan Society of Plasma Science and Nuclear Fusion Research

Keywords: neutral particle, pressure modulation, boundary plasma, microwave discharge

DOI: $10.1585 /$ pfr.5.S2075

\section{Introduction}

Stationary visible patterns associated with vortices have been observed in the HYPER-I device of the National Institute for Fusion Science, where a large volume of plasma (diameter: $300 \mathrm{~mm}$; length: $2000 \mathrm{~mm}$ ) has been produced by $2.45 \mathrm{GHz}$ microwaves with the power up to $\sim 10 \mathrm{~kW}[1]$. To investigate the properties of such the patterns, extensive plasma flow measurements have been made using directional Langmuir probes [2]. The results indicate that plasma flow or rotation can be explained by the $\boldsymbol{E} \times \boldsymbol{B}$ drift in some cases but not in other cases. Thus, laser-induced fluorescence (LIF) Doppler spectroscopy measurements have been made, resulting the importance of neutral gas particles, since the LIF measurement can be applied to either plasma ions or neutral gas particles by tuning the laser wavelength [3]. Evidence of the coupling between plasma and neutral gas particles in the bulk plasma suggests the existence of pressure modulation of neutral gas particles at the plasma boundary.

A piezoelectric transducer could offer easy measurement of neutral gas particles: in fact, piezoelectric transducers have been used to excite and detect pressure modulation due to sound waves or pseudo-waves $(>20 \mathrm{kHz})$ under a gas pressure of $\sim 10^{-3}$ Torr [4] and around a gas pressure of $\sim 10^{-1}$ Torr [5].

In this paper, we present the first measurement of the pressure modulation of neutral gas particles near the cylindrical inner wall surrounding the plasma in the frequency range of $10 \mathrm{kHz}$ and investigate the results by measuring the ion saturation currents of two Langmuir probes.

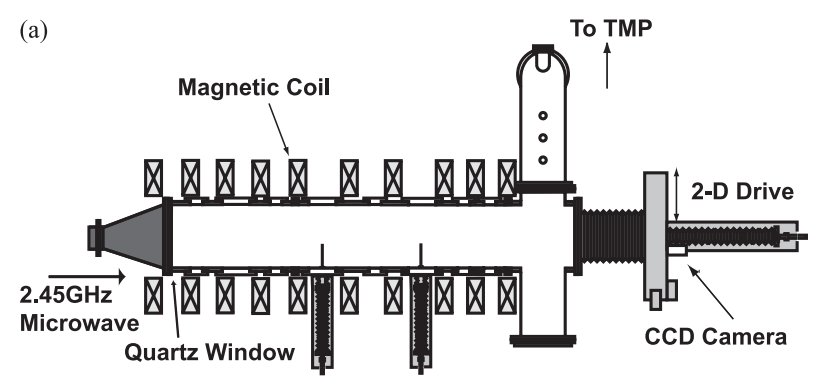

(b)

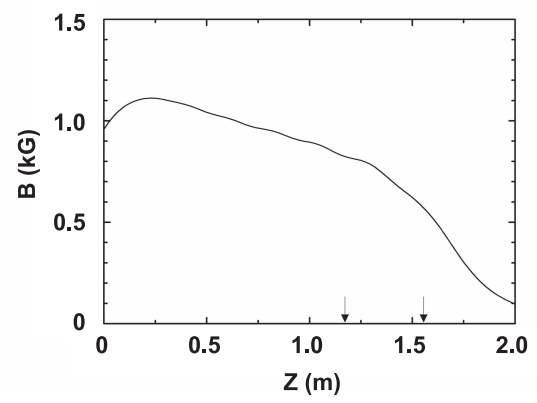

Fig. 1 (a) Sketch of HYPER-I device and (b) axial profile of magnetic field intensity on the axis.

\section{Experimental Setup}

The experiment was performed in the HYPER-I device, which has an inner diameter of $300 \mathrm{~mm}$ and a length of $2000 \mathrm{~mm}$. A sketch of the device is shown in Fig. 1 (a) and the axial profile of the magnetic field intensity on the axis is shown in Fig. 1 (b). Helium plasma was produced using $2.4 \mathrm{GHz}$ microwaves injected in the axial direction from a quartz window at $z=0 \mathrm{~mm}$. The location of the resonance surface with $\omega_{\text {ce }} / 2 \pi=2.45 \times 10^{9}$ was at $z \simeq 1060 \mathrm{~mm}$. 


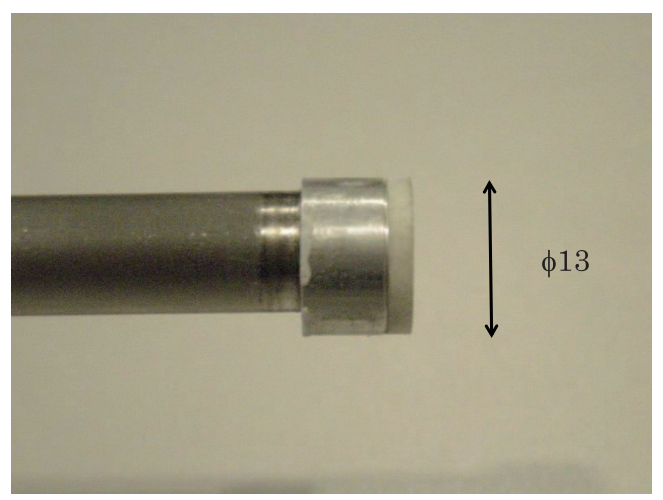

Fig. 2 Piezoelectric transducer on top of cylindrical support.

A piezoelectric transducer (FUS-300A) with an outer diameter of $13 \mathrm{~mm}$, located on the top of a cylindrical support as shown in Fig. 2, was set in the port at $z=1175 \mathrm{~mm}$ and its surface of the transducer was retracted by $10 \mathrm{~mm}$ from the inner surface of the vacuum vessel in the radial direction. The sensitivity of the transducer output is considered to be independent of frequencies in the range of $\sim 10 \mathrm{kHz}$, since its resonant frequency is $300 \mathrm{kHz}$. To measure ion saturation currents, two Langmuir probes with a bias of $-90 \mathrm{~V}$ were used. One (Probe A) was set at $z=1555 \mathrm{~mm}$ on the same side as the transducer, which can be moved in the radial direction, and the other (Probe B) at $z=1175 \mathrm{~mm}$, which was the same axial location as the transducer, and at $r=140 \mathrm{~mm}$ on the side opposite the transducer.

\section{Results}

For a microwave power of $P_{\mu}=12.5 \mathrm{~kW}$ and a helium gas pressure of $p=3.0 \times 10^{-3}$ Torr, time traces of the transducer output and the ion saturation current at $r=140 \mathrm{~mm}$ of Probe A were obtained at $t \simeq 2.0 \mathrm{~s}$ after the breakdown, as shown in Figs. 3 (a) and (b), respectively. The sampling frequency was $20 \mathrm{MHz}$. These time traces appear asymmetric about average values and are bursty in nature. Such signals are characterized by the skewness,

$$
S=\frac{1}{N} \sum_{i=1}^{N}\left(x_{i}-\langle x\rangle\right)^{3} / \sigma^{3},
$$

and the kurtosis,

$$
K=\frac{1}{N} \sum_{i=1}^{N}\left(x_{i}-\langle x\rangle\right)^{4} / \sigma^{4},
$$

where the average value $\langle x\rangle$ and the standard deviation $\sigma$ are given by

$$
\langle x\rangle=\frac{1}{N} \sum_{i=1}^{N} x_{i},
$$

and

$$
\sigma=\sqrt{\frac{1}{N} \sum_{i=1}^{N}\left(x_{i}-\langle x\rangle\right)^{2}},
$$

(a)

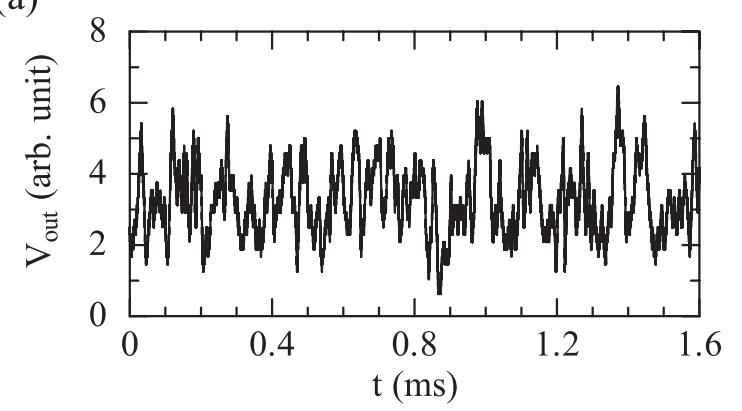

(b)

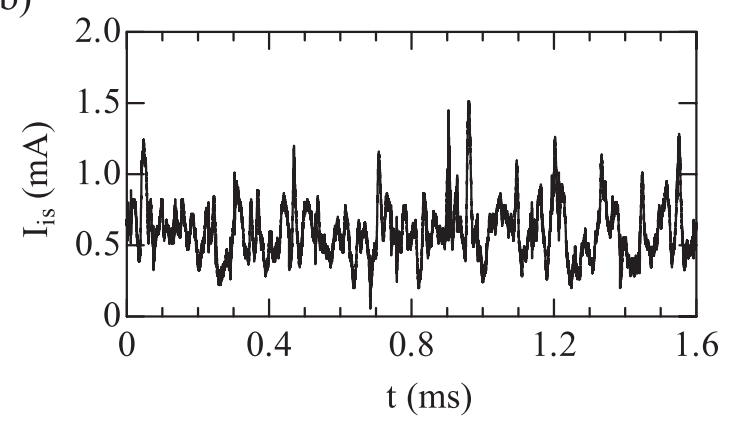

Fig. 3 (a) Time trace of transducer output $(z=1175 \mathrm{~mm})$ and (b) that of ion saturation current of Probe A $(z=$ $1555 \mathrm{~mm})$ at $r=140 \mathrm{~mm}$ for $P_{\mu}=12.5 \mathrm{~kW}$ and $p=$ $3.0 \times 10^{-3}$ Torr.

respectively [6]. In fact, $S=0.478$ and $K=2.96$ for the time trace of Fig. 3 (a), and $S=0.668$ and $K=3.97$ for that of Fig. 3 (b). Since $S=0$ and $K=3$ for a Gaussian random signal, the positive values of $S$ indicate that the time traces contain positive spikes, and the values of $K$ indicate that the magnitude of the spikes are larger in the time trace of Fig. 3 (b) than that of Fig. 3 (a). The results also indicate that the pressure modulation is not explained by a simple sinusoidal signal, which is expected if it is caused by simple sound wave, since $S=0$ and $K=1.5$ for a sinusoidal signal.

The radial profiles of the values are evaluated from the ion saturation current of Probe A $(z=1555 \mathrm{~mm})$ for $P_{\mu}=12.5 \mathrm{~kW}$ and $p=3.0 \times 10^{-3}$ Torr. The radial profiles of the average value $\left\langle I_{\text {is }}\right\rangle$, the normalized standard deviation $\sigma /\left\langle I_{\text {is }}\right\rangle$, the skewness $S$, and the kurtosis, $K$ are shown in Figs. 4 (a), (b), (c), and (d), respectively. The large values of $\sigma /\left\langle I_{\text {is }}\right\rangle$ indicate that the normalized magnitude of the fluctuation, the skewness, $S$, and the kurtosis, $K$ at the boundary layer of $r>140 \mathrm{~mm}$ increase sharply toward the vessel wall $\left(\sigma /\left\langle I_{\text {is }}\right\rangle>0.3, S>1\right.$, and $\left.K>4\right)$ and may be related to the intermittent bursty fluctuations and the convective radial transport or blob motion $[7,8]$.

We then compare the average value of the transducer output with the average value, the normalized standard deviation, and the kurtosis of the ion saturation currents for of $P_{\mu}=12 \mathrm{~kW}$ expecting that the average value of the neutral gas pressure depends on the recombination of radially transported ions at the vessel wall, and that fluctua- 
(a)

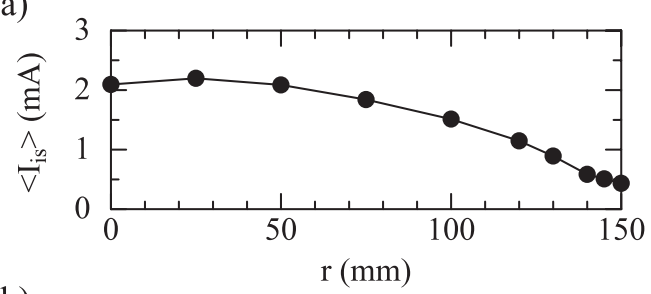

(b)

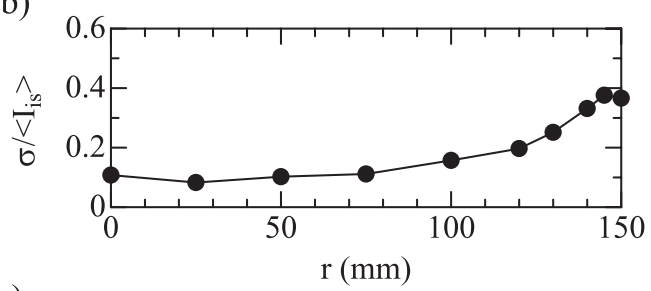

(c)

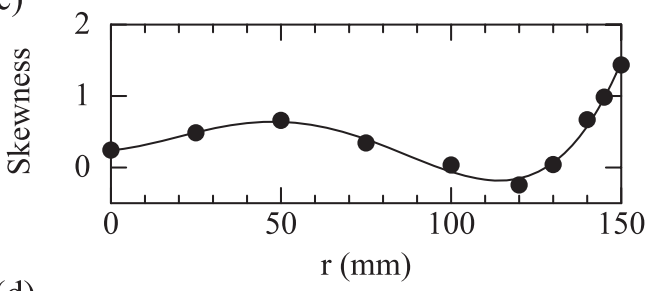

(d)

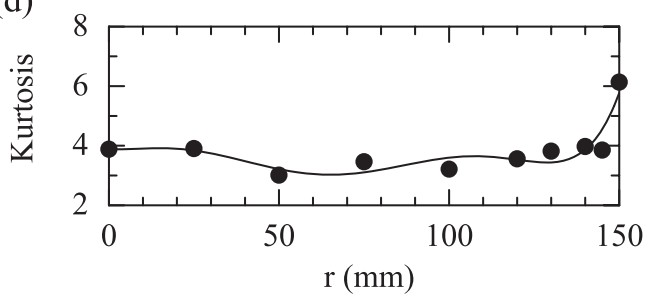

Fig. 4 Radial profiles of (a) average value, (b) normalized standard deviation, (c) skewness, and (d) kurtosis of ion saturation current of Probe A $(z=1555 \mathrm{~mm})$ for $P_{\mu}=$ $12.5 \mathrm{~kW}$ and $p=3.0 \times 10^{-3}$ Torr.

tions in the boundary plasma might cause radial ion transport. The pressure dependence of the average value of the transducer output, $\left\langle V_{\text {out }}\right\rangle$, is shown in Fig. 5 (a). The pressure dependence of the average value $\left\langle I_{\text {is }}\right\rangle$, the normalized standard deviation $\sigma /\left\langle I_{\text {is }}\right\rangle$, and the kurtosis $K$ of the ion saturation currents of Probe A $(z=1555 \mathrm{~mm}$ and $r=140 \mathrm{~mm}$ ) are shown by closed circles, and those of Probe B $(z=1175 \mathrm{~mm}$ and $r=140 \mathrm{~mm})$ by open circles in Figs. 5 (b), (c), and (d), respectively. The average value of the neutral gas pressure seems to be related to the fluctuations in the boundary plasma, but we need further studies to reach a conclusion.

The power spectra of the time traces of Figs. 3 (a) and (b) are successively evaluated; the results are shown in Figs. 6(a) and (b), respectively. The power spectrum of the ion saturation current of Probe B $(z=1175 \mathrm{~mm}$ and $r=140 \mathrm{~mm}$ ), simultaneously measured with the time traces of Figs. 3(a) and (b), is shown in Fig. 6(c). The peaks of the spectrum in Fig. 6(a), the transducer output $(z=1175 \mathrm{~mm})$, are 7,12 , and $20 \mathrm{kHz}$. The peaks of the spectrum in Fig. 6 (b), the ion saturation current of Probe $\mathrm{A}$, are 7 and $18 \mathrm{kHz}$; and the peaks of the spectrum in (a)

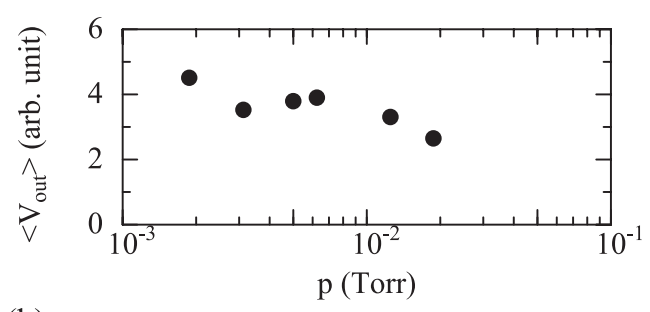

(b)

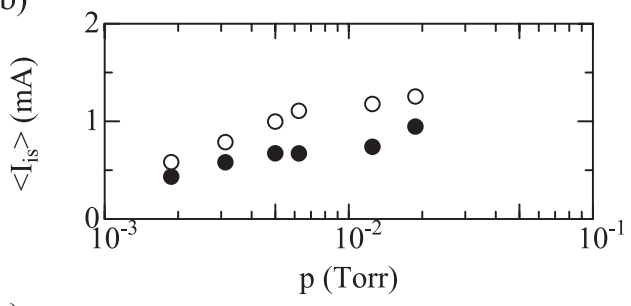

(c)

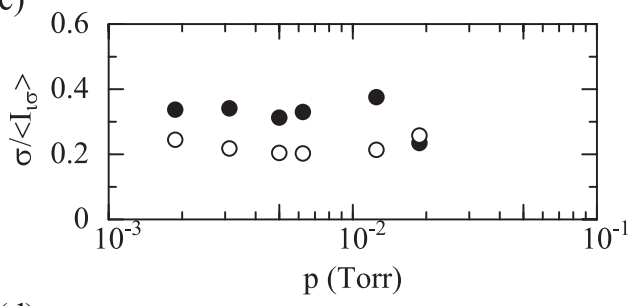

(d)

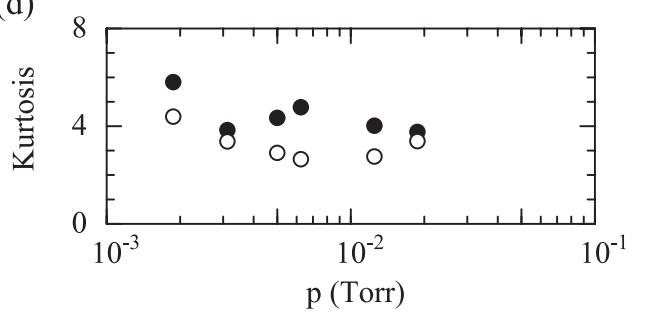

Fig. 5 (a) Pressure dependence of average value of transducer output $(z=1175 \mathrm{~mm})$; pressure dependence of (b) average value, (c) normalized standard deviation, and (d) kurtosis of ion saturation currents of Probe A $(z=1555 \mathrm{~mm}$ and $r=140 \mathrm{~mm}$ ), indicated by closed circles, and of Probe B $(z=1175 \mathrm{~mm}$ and $r=140 \mathrm{~mm})$, indicated by open circles, for $P_{\mu}=12 \mathrm{~kW}$.

Fig. 6(c), the ion saturation current of Probe B, are 7 and $11 \mathrm{kHz}$. The spectrum of the transducer output seems to be a superposition of the spectra of the ion saturation currents of Probes A and B. Since the ion saturation currents of the two probes does not represent the entire radial plasma loss, the transducer output contains components that cannot be related to the ion saturation currents of the two probes. The coherency of the peaks in the spectra of the transducer output and of the ion saturation currents of Probe A or B is not sufficiently high. The lack of sufficient coherency may be explained by the fact that the measured locations are separated by a substantial distance, and the signals are slightly bursty. Thus, one might say that the peaks of the spectra of the ion saturation currents reflect the convective radial transport or blobs, and those of the spectrum of the transducer output are caused by the recombination of the plasma convectively carried out to the wall. 
(a)

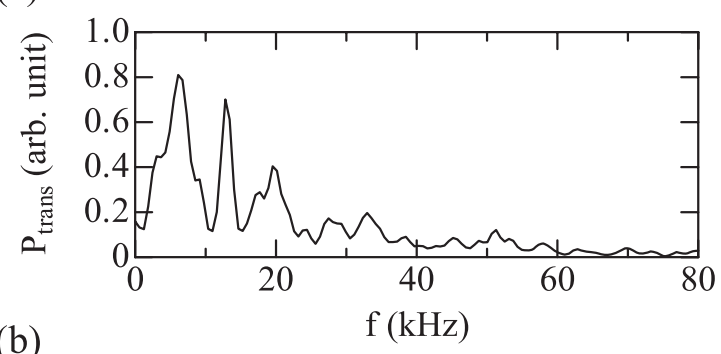

(b)

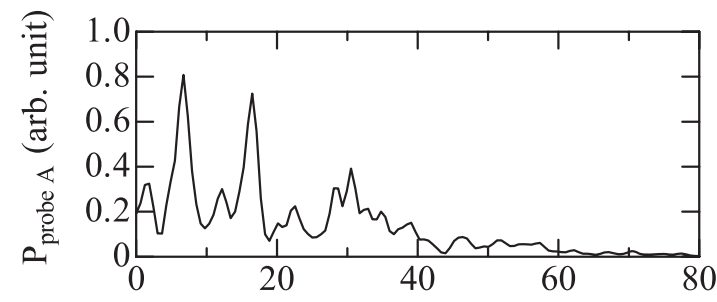

(c)

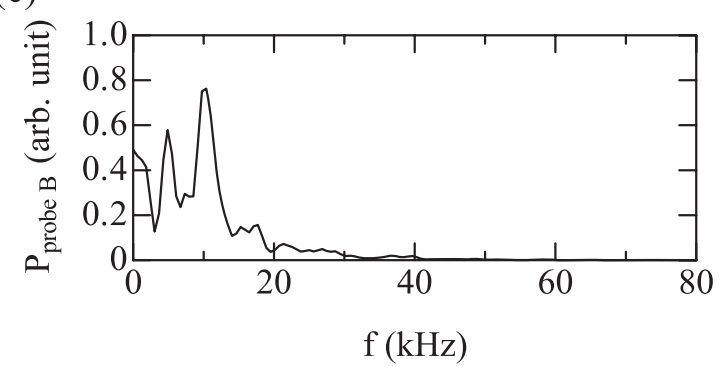

Fig. 6 Power spectra of (a) transducer output $(z=1175 \mathrm{~mm})$, (b) ion saturation current of Probe A $(z=1555 \mathrm{~mm}$ and $r=140 \mathrm{~mm}$ ) and (c) ion saturation current of Probe B $\left(z=1175 \mathrm{~mm}\right.$ and $r=140 \mathrm{~mm}$ ) for $P_{\mu}=12.5 \mathrm{~kW}$ and $p=3.0 \times 10^{-3}$ Torr.

\section{Summary}

A piezoelectric transducer was used to measure the pressure modulation of neutral gas particles near the cylin- drical inner wall $(r=150 \mathrm{~mm})$ of the HYPER-I device, which has an axial length of $2000 \mathrm{~mm}$, where helium plasma was produced by discharge using electron cyclotron resonance of $2.45 \mathrm{GHz}$ microwaves with a power of $\sim 12 \mathrm{~kW}$. The frequency of the obtained pressure modulation was in the range of $10 \mathrm{kHz}$. The time traces of the ion saturation currents at the boundary plasma of $r=140 \mathrm{~mm}$ contain intermittent positive spikes with a skewness of $S \simeq 1$ and a kurtosis of $K \simeq 4$, which may be related to convective radial transport or blob motion of the boundary plasma. The obtained peaks of the power spectrum of the transducer output might be a combination of those of the power spectra of the ion saturation currents of two Langmuir probes separated by $380 \mathrm{~mm}$ in the axial direction and by $180^{\circ}$ in the azimuthal direction at the plasma boundary of $r=140 \mathrm{~mm}$.

\section{Acknowledgment}

This work was performed under the auspices of the NIFS Collaboration Research program (NIFS09KQHP019).

[1] M. Y. Tanaka, R. Nishimoto, S. Higashi, N. Harada, T. Ohi, A. Komori and Y. Kawai, J. Phys. Soc. Jpn. 60, 1600 (1991).

[2] K. Nagaoka, A. Okamoto, S. Yoshimura and M. Y. Tanaka, J. Phys. Soc. Jpn. 70, 131 (2001).

[3] A. Okamoto, S. Yoshimura, S. Kado and M. Y. Tanaka, J. Plasma Fusion Res. 80, 1003 (2004).

[4] A. Tsushima and T. K. Tanaka, J. Phys. Soc. Jpn. 71, 1805 (2002).

[5] A. Tsushima, Jpn. J. Appl. Phys. 45, 8141 (2006).

[6] H. L. Pècseli, Fluctuations in Physical Systems (Cambridge University Press, Cambridge, 2000).

[7] N. Ohno, V. P. Budaev, K. Furuta, H. Miyoshi and S. Takamura, Contrib. Plasma Phys. 44, 222 (2004).

[8] S. I. Krasheninnikov and A. I. Smolyakov, Phys. Plasmas 10, 3020 (2003). 